Leser der MMW können sich ab sofort mit allen Fragen zur Abrechnung und Praxisführung an Helmut Walbert, Facharzt für Allgemeinmedizin, Würzburg, wenden. Sie erreichen ihn jeden Donnerstag von 13 bis 15 Uhr unter der kostenlosen Rufnummer (080o) 2379830 oder per E-Mail: w@lbert.info.
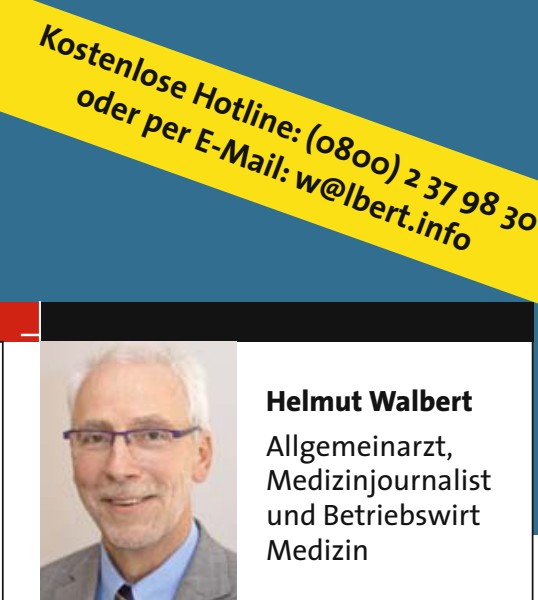

Helmut Walbert

Allgemeinarzt, Medizinjournalist und Betriebswirt Medizin

\title{
Jetzt sollen wir die postoperative Behandlung übernehmen
}

Frage von Dr. med. W.V., Allgemeinarzt, Würzburg:

Ein Patient hat von sich aus einen Orthopäden aufgesucht, wurde ambulant operiert und kam dann zur postoperativen Behandlung zu mir, weil ihm offensichtlich der Weg zum Orthopäden zu aufwendig war. Welche Leistungen können wir korrekterweise abrechnen? Der Patient hat uns eine Überweisung vom Orthopäden zur Weiter- und Mitbehandlung mitgebracht. Können wir die Versichertenpauschale, die halbe Versichertenpauschale oder müssen wir gar die Konsultationspauschale, die Gebührenordnungsposition (GOP) 01 436, berechnen?
Antwort: Sie können auf jeden Fall die Versichertenpauschale GOP O3 110 bis O3 112 abrechnen, da Sie Ihren Patienten zum ersten Mal im Quartal sehen. Wäre es ein Vertretungspatient oder käme die Überweisung von einem Arzt der gleichen Fachgruppe - also einem Allgemeinarzt -, dann käme die GOP O3 120 bis 03122 zum Ansatz.

Würde der Patient von einem Arzt der gleichen Fachgruppe lediglich zur postoperativen Behandlung als Auftrag überwiesen, dann käme die GOP 01436 infrage.

Ein Punkt, der aber in jedem Fall von Bedeutung ist: Der Operateur muss eine
Überweisung ausstellen zur postoperativen Behandlung unter Angabe des Operationen- und Prozedurenschlüssels (OPS) der durchgeführten Operation. Der Operateur dokumentiert mit der Überweisung die Beauftragung und die Tatsache, dass er selbst die Leistung nicht abrechnet.

Darüber hinaus muss eine Ausschlussbestimmung beachtet werden: Innerhalb von 21 Tagen postoperativ sind unter vielen anderen vor allem operative Leistungen nach GOP 02300 bis 02302 und die GOP 02 310, die Behandlung sekundär heilender Wunden, ausgeschlossen.

\section{Wie oft darf ich die GOP 33061 berechnen?}

Frage von Dr. med. F.-J. Z., Hausarztinternist, KVNO:

Ich rechne seit Jahren die sonografische Untersuchung der Extremitätengefäße je Extremität ab, also in der Regel zweimal je Untersuchung. Jetzt habe ich festgestellt, dass die KV im Rahmen der sachlichen und rechnerischen Richtigstellung die Gebührenordnungsposition (GOP) 33061 nur einmal pro Tag zulässt. Ist das richtig?

Antwort: Leider ja. Zum 1. Januar 2008 gab es die „geringfügige“ Änderung eines Kommas in dieser GOP! Bis 31.12.2007 gab es vor der Bestimmung „je Extremität“ ein Komma. Dieses wurde gestrichen und damit erhält die Bestimmung ,je Sitzung “ eine ausschließende Bedeutung: Seitdem ist die GOP 33061 nur einmal berechenbar. Gleichzeitig wird die Anzahl der Untersuchungsstellen je Extremität „mindestens drei Ableitungsstellen je Extremität“ zur Mindestanforderung.

Auf den ersten Blick eine unscheinbare Änderung, die offensichtlich vielen Kolleginnen und Kollegen noch gar nicht aufgefallen ist. Seit 1. Januar 2008 gilt es, die Leistung gegebenenfalls auf zwei Sitzungen zu splitten, wenn sowohl obere und untere Extremitäten untersucht werden.
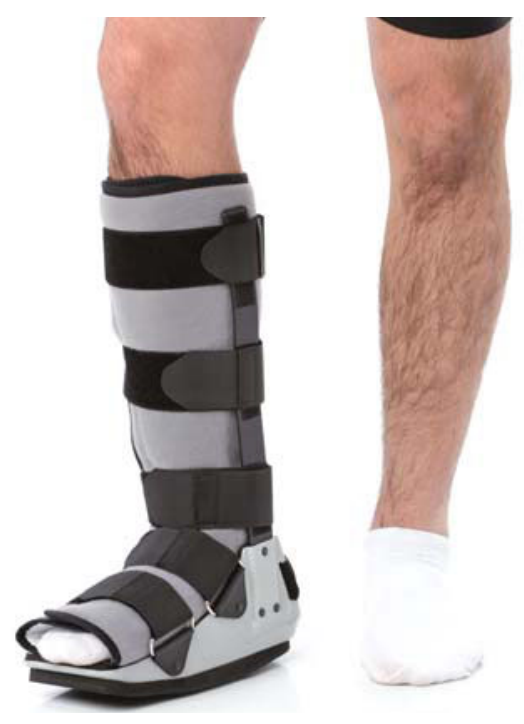

Hat der Operatuer eine Überweisung ausgestellt, kann der Allgemeinarzt weiterbehandeln. 